\title{
Effect of trans fatty acid intake on abdominal and liver fat deposition and blood lipids: a randomized trial in overweight postmenopausal women
}

\author{
NT Bendsen ${ }^{1}$, E Chabanova ${ }^{2}$, HS Thomsen ${ }^{3}$, TM Larsen $^{1}$, JW Newman ${ }^{4,5}$, S Stender ${ }^{6}$, J Dyerberg ${ }^{1}$, \\ SB Haugaard ${ }^{7}$ and A Astrup ${ }^{1}$
}

${ }^{1}$ Department of Human Nutrition, Faculty of Life Sciences, University of Copenhagen, Frederiksberg, Denmark; ${ }^{2}$ Department of Diagnostic Radiology, Copenhagen University Hospital, Copenhagen, Denmark; ${ }^{3}$ Department of Diagnostic Sciences, Faculty of Health Sciences, University of Copenhagen, Copenhagen, Denmark; ${ }^{4}$ United States Department of Agriculture, Agricultural Research Service, Western Human Nutrition Research Center, Davis, CA, USA; ${ }^{5}$ Department of Nutrition, University of California, Davis, CA, USA; ${ }^{6}$ Department of Clinical Biochemistry, Copenhagen University Hospital, Copenhagen, Denmark and ${ }^{7}$ Clinical Research Centre, Copenhagen University Hospital, Copenhagen, Denmark

Background: Intake of industrially produced trans fatty acids (TFAs) is, according to observational studies, associated with an increased risk of cardiovascular disease, but the causal mechanisms have not been fully elucidated. Besides inducing dyslipidemia, TFA intake is suspected to promote abdominal and liver fat deposition.

Objective: We examined the effect of a high intake of TFA as part of an isocaloric diet on whole-body, abdominal and hepatic fat deposition, and blood lipids in postmenopausal women.

Methods: In a 16-week double-blind parallel intervention study, 52 healthy overweight postmenopausal women were randomized to receive either partially hydrogenated soybean oil providing $15.7 \mathrm{~g}_{\text {day }}{ }^{-1}$ of TFA or a control oil with mainly oleic and palmitic acid. Before and after the intervention, body composition was assessed by dual-energy X-ray absorptiometry, abdominal fat by magnetic resonance (MR) imaging, and liver fat by ${ }^{1} \mathrm{H}$ MR spectroscopy.

Results: Compared with the control fat, TFA intake decreased plasma high-density lipoprotein (HDL)-cholesterol by $10 \%$, increased low-density lipoprotein (LDL)-cholesterol by $18 \%$ and resulted in an increased LDL/HDL-cholesterol ratio (baseline adjusted mean $(95 \% \mathrm{Cl})$ difference between diet groups $0.41(0.22 ; 0.60) ; P<0.001)$. TFA tended to increase the body fat $(0.46$ $(-0.20 ; 1.17) \mathrm{kg} ; P=0.16)$ and waist circumference $(1.1(-0.1 ; 2.4) \mathrm{cm} ; P=0.08)$ more than the control fat, whereas neither abdominal nor liver fat deposition was affected by TFA.

Conclusion: The adverse effect of dietary TFA on cardiovascular disease risk involves induction of dyslipidemia, and perhaps body fat, whereas weight gain-independent accumulation of ectopic fat could not be identified as a contributory factor during short-term intake.

Nutrition and Diabetes (2011) 1, e4; doi:10.1038/nutd.2010.4; published online 31 January 2011

Keywords: randomized clinical trial; trans fatty acids; ectopic fat; dietary intervention

\section{Introduction}

A high intake of industrially produced trans fatty acids (TFAs) has, in observational studies, consistently been shown to be associated with an increased risk of coronary heart disease. In a meta-analysis of four prospective cohort studies, it was

Correspondence: Professor A Astrup, Department of Human Nutrition, Faculty of Life Sciences, University of Copenhagen, Rolighedsvej 30, DK-1958 Frederiksberg, Denmark.

E-mail: ast@life.ku.dk

Received 30 September 2010; revised 15 November 2010; accepted 17 November 2010 estimated that that an isocaloric substitution of $2 \%$ of the total energy intake $(E \%)$ of carbohydrates with TFA corresponds to a $23 \%$ increase in the risk of cardiovascular events. ${ }^{1}$

It has been well established that TFA intake increases the ratio of total cholesterol (TC) to high-density lipoproteincholesterol (HDL-C) in the blood. A recent meta-analysis estimated that an absolute increase of $2 \mathrm{E} \%$ in the intake of TFA is associated with an increase in the TC/HDL-C ratio of $\sim 0.1$ unit, when replacing cis monounsaturated fatty acids (MUFAs). ${ }^{2}$ An increase of this size is estimated to increase the risk of coronary heart disease by $\sim 5 \%,{ }^{3}$ suggesting that TFA exerts harmful effects beyond those affecting cholesterol 
concentrations. Even when taking into account the possible unfavorable effects of TFA on lipoprotein (a), triglycerides, apolipoprotein (Apo) B/ApoAI ratio and C-reactive protein, a considerable explanatory gap persists. ${ }^{2}$ We hypothesize that this gap might be partly explained by adverse effects of TFA on body fat deposition.

Abdominal fat deposition is an established risk factor for cardiovascular disease, and emerging evidence suggests that liver fat should be considered to be one as well. ${ }^{4}$ TFA intake was positively associated with a modest increase in waist circumference during 9 years of follow-up in the Health Professional's Follow-up Study, even after adjustment for body mass index, ${ }^{5}$ and with weight change during 8 years of follow-up in the Nurses' Health Study. ${ }^{6}$ Moreover, a recent intervention study in non-human primates suggested that TFA might stimulate visceral fat deposition irrespective of weight gain when consumed in considerable amounts over a long period of time, ${ }^{7}$ and rodent studies have shown that a high TFA intake may lead to increased deposition of fat in the liver. ${ }^{8,9}$ In humans, it has yet to be examined whether TFA intake affects abdominal or liver fat deposition.

We conducted a dietary intervention study with the objective of examining the effect of a high intake of industrially produced TFA on lipid deposition in liver and in the abdominal region in TFA-depleted overweight postmenopausal women. Also, we wished to confirm previous findings of the effect of TFA on blood lipids.

\section{Subjects and methods}

The present work examined the effect of a high intake of TFA on risk markers for cardiovascular disease, with the primary end points being low-density lipoprotein-cholesterol (LDLC)/HDL-C ratio and liver fat deposition.

\section{Study design}

We conducted a 16-week double-blind, parallel dietary intervention study, in which 52 women were randomized to two test diets stratified by waist circumference. The cut-off waist circumference was set at $96 \mathrm{~cm}$ for separation into strata. For each stratum a randomization sequence with random permuted blocks of four was computer generated by a third party prior to initiating the randomization. A coding list was given to the department's kitchen staff. The investigators responsible for seeing the study subjects allocated the next available number on entry into the trial, and each woman collected her experimental diet directly from the kitchen. Thereby the code was kept undisclosed for the investigators until after study completion.

All study personnel and participants were blinded to the treatment assignment for the duration of the study. Information about which diet was assigned to which group was not disclosed to the researcher responsible for the statistical analyses before completion of the analyses.
The women visited the department for four examinations during the study: at screening (1-8 weeks prior to baseline), baseline (week 0), mid-intervention (week 8) and at the end of treatment (week 16). In addition, the subjects attended the department for control weighing at weeks 4 and 12. The habitual physical activity level during the last year was assessed at baseline using the questionnaire by Baecke et al. ${ }^{10}$ and subjects were instructed to maintain their habitual activity level throughout the dietary intervention period. Subsequent to the dietary intervention period, the subjects participated in a 12-week dietary weight loss program, after which they came in for follow-up examinations. Data from these examinations will be reported elsewhere.

Subjects attended all visits after an overnight fast of at least $10 \mathrm{~h}$. They were requested to avoid vigorous exercise and alcohol consumption on the day before and on the day of examinations at weeks 0,8 and 16. Also, subjects were instructed to consume similar evening meals on the evening before the visits at weeks 0 and 16 .

\section{Subjects}

The participating women were recruited from Copenhagen and the surrounding areas by advertisements posted on university web pages and in the local media. Subject inclusion criteria were as follows: being healthy; being moderately overweight (body mass index between 25 and $32 \mathrm{~kg} \mathrm{~m}^{-2}$ ); having a waist circumference $>80 \mathrm{~cm}$; been postmenopausal (self-reported) for the last 12 months; and being aged 45-70 years, extremes included. The exclusion criteria were the following: having diabetes or other chronic diseases (active cancer, psychiatric disorders, moderateto-severe rheumatologic disorders, active gastrointestinal disorders, chronic lung disease); having a history of cardiovascular disease; been smoking (currently and within 6 months prior to screening); having hypertension ( $>160$ / $100 \mathrm{~mm} \mathrm{Hg}$ ); having fasting plasma triglycerides $>3 \mathrm{mmoll}^{-1}$; having fasting plasma LDL-C $>6 \mathrm{mmoll}^{-1}$; having fasting plasma glucose $>7 \mathrm{mmol}^{-1}$; using hormones or anti-hypertensive, anti-lipidemic or anti-cholesterolemic drugs; having had weight changes $>3 \mathrm{~kg}$ for 2 months prior to screening; participating in strenuous physical activity $>10 \mathrm{~h}$ per week; and having abnormalities in routine biochemical and hematological tests. The subjects were not allowed to donate blood during the study and 3 months prior to screening, or to use fish oil as dietary supplements during the study and 1 month prior to commencement.

To assess how adversely the metabolism of the intervention group was affected by their elevated weight, the study included 19 lean reference subjects (body mass index = $19-24 \mathrm{~kg} \mathrm{~m}^{-2}$; waist circumference $\leqslant 80 \mathrm{~cm}$ ) who underwent baseline examinations only. These reference subjects fulfilled all other inclusion and exclusion criteria as the intervention subjects.

The subjects were given both verbal and written information, whereupon all gave written consent. The study was 
carried out at the Department of Human Nutrition, Faculty of Life Sciences, University of Copenhagen, Frederiksberg, Denmark, between April 2008 and March 2009 and was approved by the Municipal Ethical Committee of The Capital Region of Denmark in accordance with the Helsinki-II declaration (H-B_2007-089). Subjects received 900 US\$ as compensation on completion of all the tests. Lean reference subjects received $\sim 500$ US\$. The trial was registered at clinicaltrials.gov as NCT00655902.

\section{Study diets}

In this study, the physiological effect of consuming $15.7 \mathrm{~g} \mathrm{day}^{-1}$ of industrially produced TFA from $26 \mathrm{~g} \mathrm{day}^{-1}$ partially hydrogenated soybean oil was assessed, and a $50 / 50 \%$ mix of palm oil and high-oleic sunflower oil was used as the control. Both test fats were supplied by AarhusKarlshamn, Aarhus C, Denmark. The fatty acid (FA) composition of the two test fats was analyzed chemically as described below for red blood cell (RBC) FA composition. The two fats mainly differed in the content of TFA, palmitic (16:0), oleic (cis18:1n-9) and linoleic (cis, cis18:2n-6) acid (Table 1). The test fats were incorporated into carrot bread rolls, baked in the department's metabolic kitchen. The study participants were instructed to consume two bread rolls per day, providing $26 \mathrm{~g} \mathrm{day}^{-1}$ of test fat and a total of $2500 \mathrm{~kJ}$ (approximately $7 \mathrm{E} \%$ protein, $41 \mathrm{E} \%$ fat and $51 \mathrm{E} \%$

Table 1 Fatty acid composition of the two test fats

\begin{tabular}{|c|c|c|}
\hline Fatty acid (w/w\%) & Control fat & TFA fat \\
\hline C14:0 & 0.7 & 0.2 \\
\hline C16:0 & 26.7 & 12.7 \\
\hline C16:1-trans $(n-7)^{a}$ & $<0.1$ & $<0.1$ \\
\hline C16:1-cis (n-7) & $<0.1$ & $<0.1$ \\
\hline C17:0 & $<0.1$ & 0.1 \\
\hline C18:0 & 3.8 & 6.2 \\
\hline Total C18:1-trans & $<0.7$ & 59.0 \\
\hline trans $(n-12) /$ trans $(n-11) /$ trans $(n-10)$ & $<0.1$ & 9.6 \\
\hline trans $(\mathrm{n}-9)$ & $<0.1$ & 12.7 \\
\hline trans $(\mathrm{n}-8)$ & $<0.1$ & 12.7 \\
\hline trans $(\mathrm{n}-7)$ & $<0.1$ & 10.7 \\
\hline trans $(\mathrm{n}-6)$ & $<0.1$ & 7.8 \\
\hline trans $(\mathrm{n}-5)$ & $<0.1$ & 3.9 \\
\hline trans $(\mathrm{n}-4)$ & $<0.1$ & 1.6 \\
\hline Total C18:1-cis & 61.4 & 19.6 \\
\hline cis $(\mathrm{n}-12) / \operatorname{cis}(\mathrm{n}-11) / \operatorname{cis}(\mathrm{n}-10)$ & $<0.1$ & 2.9 \\
\hline $\operatorname{cis}(\mathrm{n}-9)$ & 60.2 & 6.8 \\
\hline cis $(\mathrm{n}-8)$ & $<0.1$ & 3.2 \\
\hline $\operatorname{cis}(\mathrm{n}-7)$ & 0.6 & 3.4 \\
\hline $\operatorname{cis}(\mathrm{n}-6)$ & 0.6 & 3.2 \\
\hline C18:2-trans, trans (n-6) & $<0.1$ & 1.4 \\
\hline C18:2-cis, cis $(\mathrm{n}-6)$ & 6.6 & 0.2 \\
\hline $\mathrm{C} 20: 0$ & 0.4 & 0.4 \\
\hline $\mathrm{C} 22: 0$ & 0.4 & 0.3 \\
\hline Total trans fatty acids & $<0.7$ & 60.4 \\
\hline
\end{tabular}

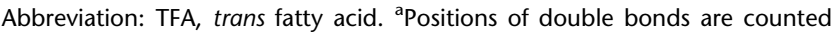
from the methyl end of the fatty acid. carbohydrate, calculated using the Dankost 3000 dietary assessment software (Danish Catering Center, Herlev, Denmark)). Frozen rolls were handed out to the women every 1-4 weeks from the department for consumption at home.

The intervention was not intended to promote weight gain; hence, a clinical dietician instructed the participants how to let the bread rolls iso-calorically substitute food items from their habitual diets. The subjects were weighed every 4 weeks during the dietary intervention. They were offered an additional dietary session if their weight increased more than $2 \mathrm{~kg}$ from baseline; three subjects from the TFA group and two from the control group accepted the offer.

\section{Compliance}

At baseline the study subjects were handed a diary in which they were to report whether or not both test rolls were consumed each day. Also, they were to weigh back and report the amounts not eaten. As an objective compliance measure, the incorporation of FA into phospholipids of RBCs was assessed at weeks 0,8 and 16 . For determination of FA composition, saline-washed RBCs were spiked with tri-heptadecenoylglyceride, solubilized in 9:1 methanol/ toluene and centrifuged. FA methyl esters in supernatant aliquots were prepared by sequential treatment with anhydrous methanolic sodium methoxide and methanolic hydrochloric acid. ${ }^{11}$ FA methyl esters were back-extracted into hexane, enriched with methyl tricosanoate (23:0) as an internal standard, and quantified by gas chromatography on high-polarity cyano-silica columns and a flame ionization detector (see also Supplementary Methods).

Owing to co-chromatography of interference with variable relative retention times on the two chromatographic systems used during this study, an accurate quantitative assessment of TFA concentrations could unfortunately not be made. However, a qualitative ranking of the presence and abundance of the TFA profile relative to cis18:1n-7 was possible (Supplementary Figure 1).

\section{Dietary intake}

The dietary intake was assessed by 3-day weighed food records before the intervention and in the last week of the intervention. The records were coded, and the energy intake and macronutrient composition were calculated by using a national food database (National Food Agency, Søborg, Denmark $^{12}$ ) with the Dankost 3000 dietary assessment software (Danish Catering Center).

\section{Anthropometrics and blood pressure}

Body weight and waist and hip circumference were measured at all visits. Body weight was measured in kilograms with one decimal by a Lindeltronic 8000 scale (Samhall Lavi AB, Kristianstad, Sweden). Height was assessed at screening to the nearest $0.5 \mathrm{~cm}$ using a Seca stadiometer (Hultafors, Sweden). Waist circumference was measured at the midpoint 
between the lower part of the last rib and the top of the hip, and hip circumference at the maximal width over the greater trochanters.

Blood pressure measurements were performed in the seated position after $10 \mathrm{~min}$ of rest, with an automatically inflated cuff (UA-787, A \& D, Saitama, Japan) at weeks 0, 8 and 16.

Body composition was measured by dual-energy X-ray absorptiometry (DXA) using a GE Lunar Prodigy Advance DXA scanner (GE Medical Systems Lunar, Madison, WI, USA). A diagonal line passing through the middle of the femoral neck separated the lower-body and trunkal regions for determination of trunk fat as percentage of total tissue mass.

\section{Magnetic resonance scans}

We assessed total-, subcutaneous- and intra-abdominal adipose tissue (AAT) volumes by magnetic resonance (MR) imaging and measured the lipid content of the liver by ${ }^{1} \mathrm{H}$ MR spectroscopy. MR examinations were performed on all subjects at weeks 0 and 16 one to three days after the other examinations (but on a similar day and time of day for each subject at both visits). The subjects were instructed to refrain from alcohol consumption and vigorous physical activity for $24 \mathrm{~h}$ prior to each scan. At the baseline visit subjects recorded all food and drinks consumed the evening before the scan and on the morning of the day of the scan. At the subsequent visit this intake was to be replicated.

All MR measurements were performed using Achieva 3.0 T MR imaging system (Philips Medical Systems, Best, The Netherlands) and a sense cardiac coil. At the beginning of the MR examination, T2w TSE coronal and axial slices through the abdomen were acquired for positioning the spectroscopy volumes of interest. The spectroscopy volume of interest $(11 \mathrm{~mm} \times 11 \mathrm{~mm})$ was positioned within the liver, avoiding major blood vessels and intrahepatic bile ducts. A single-voxel PRESS sequence with respiratory triggering was used for acquisition. The MR scanner's software was used to fit the acquired spectrum to the relative content of water and lipid. Thereby, hepatic lipid content was expressed as lipid relative to water $(\%) .{ }^{13}$

A fast T1w TFE MR sequence in transverse plane was used to obtain data for estimating the adipose tissue volumes. A transverse slice with $10 \mathrm{~mm}$ thickness was acquired for all subjects in the middle of the third lumbar vertebra (L3). Image analysis to determine the total and intra-AAT fat volumes was carried out on a Philips ViewForum workstation using the 'segmentation tool' in 'volume analysis'.

\section{Blood sampling and analysis}

Fasting blood was sampled from an antecubital vein after minimum $10 \mathrm{~min}$ of rest. Routine hematological and biochemical analyses including blood lipids were performed at the Department of Clinical Biochemistry, Gentofte University Hospital, Denmark. Fasting plasma TC, HDL-C and triglycerides were measured by dry chemistry slides on Vitros 5.1 FS (Johnson \& Johnson, Rochester, NY, USA). The CV\% values were 4.4, 6.0 and 9.6 for TC, HDL-C and triglycerides, respectively. All tests were performed in accordance with ISO 15189 accreditation. Plasma LDL-C concentration was estimated by the Friedewald equation. ${ }^{14}$

\section{Statistical analyses}

The study size was estimated based on the effect of TFA on the LDL-C/HDL-C ratio. Based on data from two metaanalyses, we assumed a within-subject s.d. of 0.6 for this ratio. ${ }^{1,15}$ We estimated that a minimum of 48 subjects was required to detect an absolute difference of 0.5 in the LDL-C/ HDL-C ratio between diet groups with a statistical power of $80 \%$ and a two-sided significance level of 5\%. Assuming an s.d. for liver fat of $6.5 \%$ in moderately obese women, ${ }^{16} \mathrm{a}$ difference between diet groups of approximately 6\% point liver fat could be detected with at a statistical power of $80 \%$, a two-sided significance level of 5\% and 24 expected completers in each group. To allow for an estimated $10 \%$ dropout rate, 52 participants were recruited.

Data were analyzed using Statistic Analysis Package, SAS version 9.1 (SAS Institute, Cary, NC, USA). The primary analysis included participants who completed the intervention $(n=49)$. The statistical significance level was defined as $P<0.05$.

Baseline values for the overweight intervention subjects were compared with values for the lean reference subjects by unpaired $t$-tests or Kruskal-Wallis tests for skewed data.

Analysis of covariance was used to assess the baselineadjusted difference between diet groups for variables measured at weeks 0 and 16 , that is, the baseline value was included as a covariate. For variables measured at weeks 0,4 , 8,12 and 16 and weeks 0,8 and 16, respectively, a mixed model of repeated measures examining the effect of diet and time (weeks 4, 8, 12 and 16) and their interactions was applied, again with the baseline value as a covariate, and with 'subject' treated as a random effect. Variance homogeneity and normality were investigated by residual plots, histograms and Shapiro-Wilk's test, and data were log transformed when needed. Analyses were performed with and without adjustment for changes in body weight.

\section{Results}

In all, 49 subjects ( 24 in the TFA group and 25 in the control group) completed the intervention (Figure 1). One subject withdrew immediately after baseline examinations (she felt medicalized by the study) and two subjects dropped out after a few weeks owing to lack of time. Baseline characteristics of the subjects in the two diet groups were comparable and the lean reference subjects were adequately matched for age and height (Table 2). 


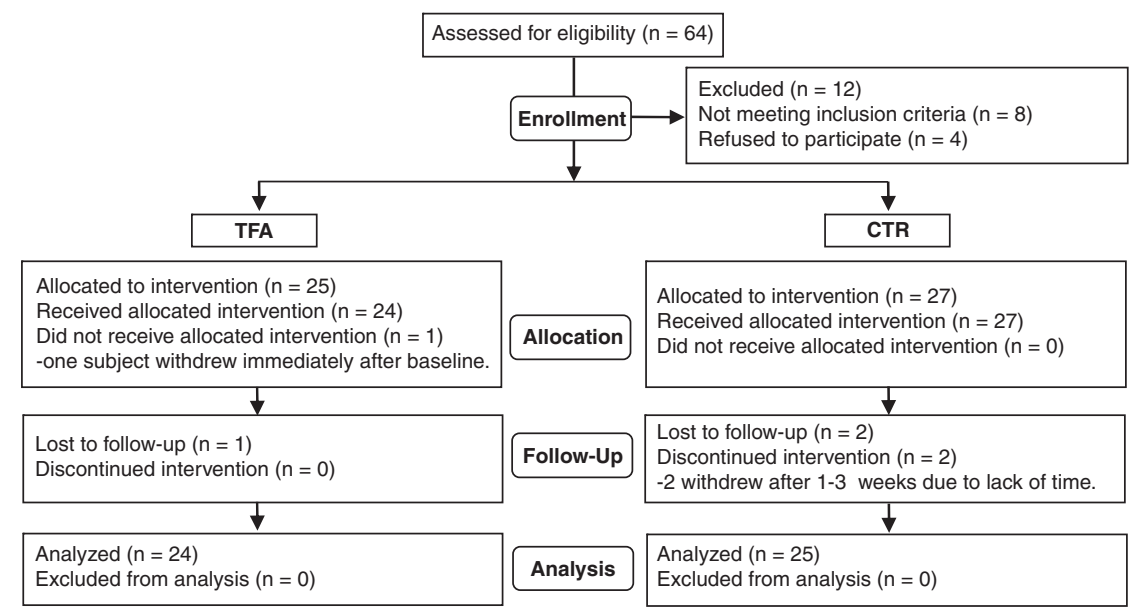

Figure 1 CONSORT flow diagram displaying subjects recruited into the dietary intervention. Subjects were randomly assigned into two diet groups: a trans fatty acid (TFA) group and a control (CTR) group.

Table 2 Baseline characteristics for subjects in the TFA $(n=24)$ and control $(n=25)$ groups and for lean reference subjects $(n=19)^{\mathrm{a}}$

\begin{tabular}{|c|c|c|c|c|}
\hline & TFA & Control & $\begin{array}{c}\text { Lean reference } \\
\text { subjects }\end{array}$ & $\mathrm{P}^{\mathrm{b}}$ \\
\hline Age (years) & $58.5 \pm 4.6$ & $58.8 \pm 5.5$ & $60.1 \pm 5.9$ & NS \\
\hline Height $(\mathrm{cm})$ & $165.3 \pm 5.5$ & $166.9 \pm 5.2$ & $166.7 \pm 4.3$ & NS \\
\hline Weight (kg) & $78.7 \pm 7.1$ & $78.4 \pm 8.6$ & $59.4 \pm 4.8$ & $<0.001$ \\
\hline BMI $\left(\mathrm{kg} \mathrm{m}^{-2}\right)$ & $28.8 \pm 1.7$ & $28.1 \pm 2.2$ & $21.3 \pm 1.4$ & $<0.001$ \\
\hline Waist circumference $(\mathrm{cm})$ & $97.1 \pm 7.3$ & $95.5 \pm 6.8$ & $74.9 \pm 3.8$ & $<0.001$ \\
\hline \multicolumn{5}{|l|}{ Blood pressure } \\
\hline Systolic (mm Hg) & $118 \pm 12$ & $118 \pm 11$ & $120 \pm 14$ & NS \\
\hline Diastolic $(\mathrm{mm} \mathrm{Hg})$ & $81 \pm 8$ & $82 \pm 6$ & $80 \pm 10$ & NS \\
\hline Physical activity index ${ }^{10}$ & $7.5 \pm 1.4$ & $7.9 \pm 1.6$ & $8.6 \pm 1.4$ & 0.02 \\
\hline
\end{tabular}

Abbreviations: NS, not significant; TFA, trans fatty acid. ${ }^{a}$ Values are means \pm s.d., completers only. ${ }^{b} p$ value refers to difference between overweight intervention subjects (diet groups combined) and lean reference subjects by unpaired $t$-tests $(P>0.05)$.

\section{Compliance with the study diets}

The test diets provided on average $28 \%$ of the subjects' energy requirements. Self-reported compliance assessed using study diaries showed that $98 \%$ of all test bread rolls were consumed, with no difference between diet groups. The presence of elevated trans 18:1 residues in RBC phospholipids determined by a gas chromatograph/flame ionization detector was used as an objective compliance measure. Owing to an analytical interference, accurate quantification of trans18:1 isomers was not possible. Instead, a qualitative intensity scale was established and used to assign a value of TFA enrichment to each sample (Supplementary Figure 1). These results were transformed to ranks and showed that all subjects in the TFA group had elevated trans 18:1 residue levels at both 8 and 16 weeks of intervention, whereas the control subjects did not (Figure 2). The intervention

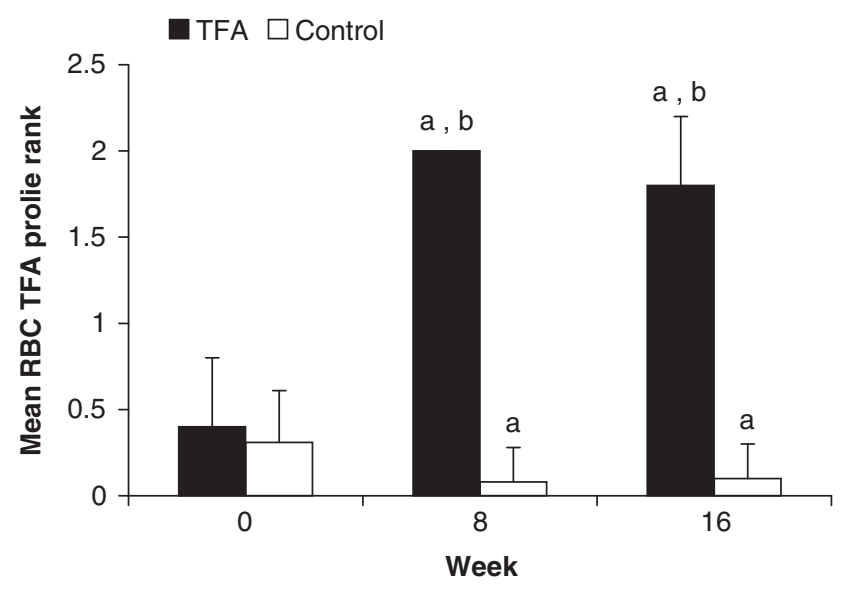

Figure 2 Semi-quantitative assessment of trans fatty acids (TFAs) in red blood cell (RBC) phospholipids assessed by gas chromatography. Based on semi-quantitative assessments of RBC trans 18:1 profiles, including the scores $0,0.5,1,1.5$ and 2 (where 2 signifies full enrichment) (Supplementary Figure 1), maximal TFA enrichment was observed by week 8 and was generally sustained at week 16 in the TFA group $(n=24)$, whereas the subjects in the control group experienced no TFA enrichment $(n=25)$. Changes from baseline TFA profiles were assessed by repeated-measures ANOVA. Owing to the non-normal distribution of the semi-quantitative data, the analyses were

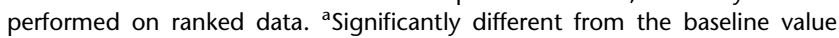
$(P<0.001)$. ${ }^{b}$ Significantly different from the control group $(P<0.001)$.

modestly decreased the relative abundance of saturated fatty acids (SFAs) and increased that of polyunsaturated fatty acids (PUFAs) in the RBCs of both diet groups after 16 weeks. Oleic acid increased in the control group only (Supplementary Table 1).

Dietary intake

The energy intake in the last week of the intervention (representing the intake during the dietary period) was not 

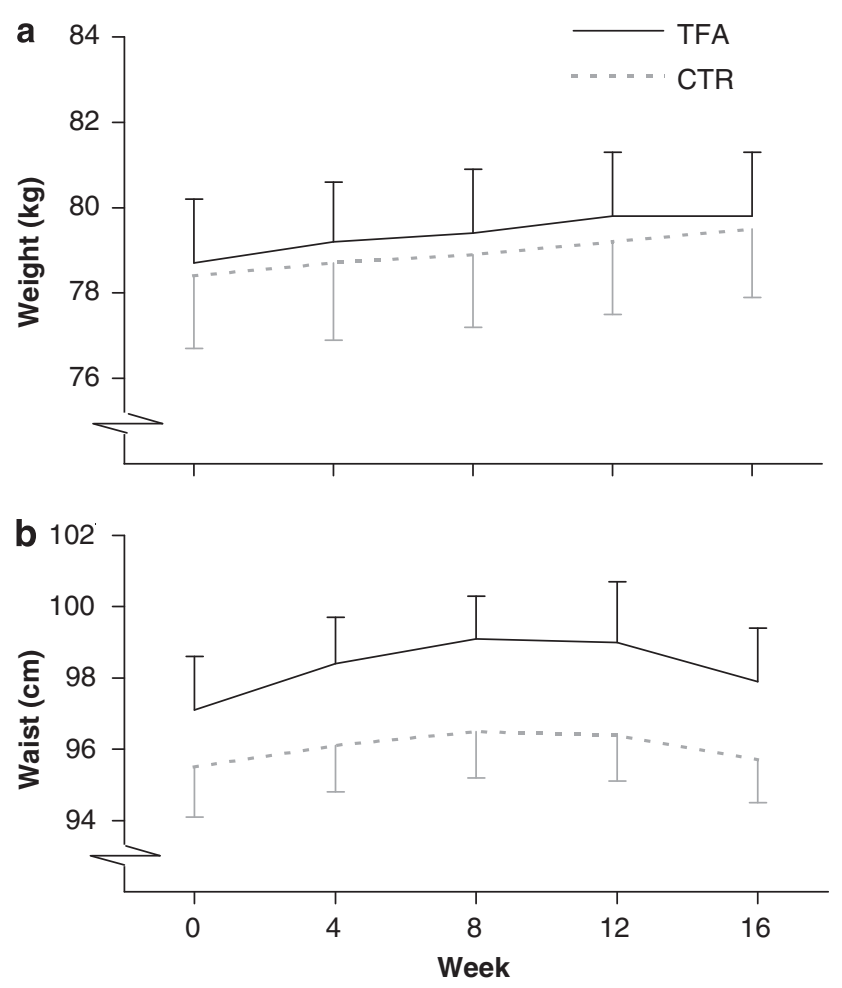

Figure 3 Development in mean ( \pm s.e.m.) body weight (a) and waist circumference (b) in the trans fat (TFA; $n=24)$ and control (CTR; $n=25)$ groups during the 16-week dietary intervention. Baseline values were not significantly different (unpaired $t$-test, $P>0.05$ ). Note that the $y$-axes have been truncated. In spite of the isocaloric study design, body weight increased significantly in both diet groups ( $P=0.002$ for effect of time in repeatedmeasures analysis of covariance testing for interaction between diet group and time (weeks $4,8,12,16$ ) with week 0 as a covariate; there was no significant interaction between diet and time, nor effect on diet). Waist circumference tended to increase more in the TFA group ( $P=0.08$ for effect of diet; there was no significant interaction between diet and time, nor effect of time).

different between diet groups (Supplementary Table 2). The only significant dietary differences between diet groups during the intervention were the contributions of energy from MUFA and TFA, indicating that the diets were overall comparable apart from the FA composition. TFA constituted $7 \%$ of the energy intake in the TFA group.

\section{Anthropometrics}

Contrary to the intention, body weight increased slowly but steadily in both diet groups during the dietary intervention, with no difference between groups $(P=0.002$ for effect of time) (Figure 3 ). At week 16, mean ( \pm s.e.m.) weight changes from baseline in the TFA and control group were $1.1 \pm 0.2$ and $1.1 \pm 0.3 \mathrm{~kg}$, respectively. Waist circumference tended to increase more in the TFA group after adjustment for baseline values (mean difference between diets $1.1 \mathrm{~cm}$ (95\% CI: -0.1 to $2.4 \mathrm{~cm}) ; P=0.08$ ); adjusting for weight change had no effect on this estimate (Figure 3 ). There was no difference between groups in hip circumference (data not shown).

\section{Fat deposition}

The changes in whole body and trunk fat mass assessed by DXA were in line with the trend for waist circumference. The fat gain was numerically greater in the TFA group, although not significantly so when compared with controls (Table 3). The baseline adjusted mean difference in body fat between diets at week 16 was $0.46 \mathrm{~kg}$ (95\% CI: -0.20 to $1.17 \mathrm{~kg}$; $P=0.16)$.

Owing to technical reasons, the liver fat was obtained by ${ }^{1} \mathrm{H}$ MR spectroscopy only in 23 subjects of each diet group, and fat distribution was obtained by MR imaging in 19 subjects of each diet group. The dietary intervention did not significantly affect the liver fat. The between-subject variability for liver fat changes was large (Figure 4), and thereby the estimate of the liver fat concentration being 7\% lower in the TFA group at week 16 compared with controls, came with a wide confidence interval (95\% CI: -61 to $120 \%$; $P=0.87$; Table 3). According to MR imaging, the dietary intervention affected neither total-, subcutaneous-, intraAAT nor the ratio of intra- to subcutaneous AAT (Table 3). As expected, the liver fat percentage as well as the subcutaneous- and intra-AAT volumes was significantly greater in overweight subjects compared with lean reference subjects.

\section{Plasma lipids}

TFA adversely affected blood cholesterol by increasing LDL-C and decreasing HDL-C (Table 4). Hereby, the ratio between the two was significantly greater in the TFA diet group at both weeks 8 and 16 compared with the control group (baseline adjusted mean (95\% CI) difference between diets 0.41 ( 0.22 to 0.60$) ; P<0.001)$. Accordingly, the TC/HDL-C ratio was also significantly increased by TFA (difference between diets $0.45(0.24$ to 0.65$) ; P<0.001)$. There was no effect of diet on fasting plasma triglycerides.

\section{Safety assessments}

Blood pressure was stable in both diet groups during the entire study period (data not shown). Hematological (hemoglobin, thrombocytes, leucocytes and erythrocytes) and biochemical (albumin, bilirubin, creatinine, sodium, and potassium) blood parameters did not change during the course of the study, nor did plasma liver enzymes (alanine aminotransferase, aspartate aminotransferase and alkaline phosphatase; data not shown).

\section{Discussion}

The present study aimed at decreasing the explanatory gap between the harmful effects of industrially produced dietary TFA seen in observational studies and the confirmed effects on individual risk markers for cardiovascular disease. Owing to the implementation of the Danish legislation, which went into force in 2003 and practically eliminated industrially 
Table 3 Body composition and abdominal fat deposition in the TFA and control groups and in lean reference subjects ${ }^{a}$

\begin{tabular}{|c|c|c|c|}
\hline & Week $0^{\mathrm{b}}$ & Week 16 & $\begin{array}{l}\text { ANCOVA } \\
\mathrm{P}_{\text {diet }}\end{array}$ \\
\hline Fat mass $(\mathrm{kg})$ & & & 0.16 \\
\hline TFA $(n=24)$ & $33.4 \pm 1.0$ & $34.6 \pm 1.1$ & \\
\hline Control $(n=25)$ & $31.8 \pm 1.1$ & $32.7 \pm 1.0$ & \\
\hline $\begin{array}{l}\text { Lean reference subjects } \\
(n=19)\end{array}$ & $16.8 \pm 0.8^{d}$ & NA & \\
\hline Fat-free mass (kg) & & & 0.20 \\
\hline TFA $(n=24)$ & $45.6 \pm 0.7$ & $45.6 \pm 0.8$ & \\
\hline Control $(n=25)$ & $47.0 \pm 1.0$ & $47.3 \pm 0.9$ & \\
\hline $\begin{array}{l}\text { Lean reference subjects } \\
(n=19)\end{array}$ & $43.3 \pm 0.6^{d}$ & NA & \\
\hline Whole body fat (\%) & & & 0.12 \\
\hline TFA $(n=24)$ & $42.1 \pm 0.7$ & $43.0 \pm 0.7$ & \\
\hline Control $(n=25)$ & $40.2 \pm 0.9$ & $40.7 \pm 0.8$ & \\
\hline $\begin{array}{l}\text { Lean reference subjects } \\
(n=19)\end{array}$ & $27.8 \pm 0.9^{d}$ & NA & \\
\hline Trunk fat (\%) & & & 0.17 \\
\hline TFA $(n=24)$ & $43.2 \pm 0.9$ & $44.2 \pm 0.8$ & \\
\hline Control $(n=25)$ & $41.3 \pm 1.0$ & $42.0 \pm 0.9$ & \\
\hline $\begin{array}{l}\text { Lean reference subjects } \\
(n=19)\end{array}$ & $25.5 \pm 1.1^{\mathrm{d}}$ & NA & \\
\hline Total AAT $\left(\mathrm{cm}^{3}\right)^{\mathrm{e}}$ & & & 0.85 \\
\hline TFA $(n=19)$ & $446 \pm 20$ & $458 \pm 20$ & \\
\hline Control $(n=19)$ & $390 \pm 21$ & $407 \pm 21$ & \\
\hline $\begin{array}{l}\text { Lean reference subjects } \\
(n=19)\end{array}$ & $138 \pm 10^{d}$ & NA & \\
\hline Subcutaneous $A A T\left(\mathrm{~cm}^{3}\right)^{\mathrm{e}}$ & & & 0.46 \\
\hline TFA $(n=19)$ & $313 \pm 15$ & $317 \pm 15$ & \\
\hline Control $(n=19)$ & $255 \pm 16$ & $261 \pm 14$ & \\
\hline $\begin{array}{l}\text { Lean reference subjects } \\
(n=19)\end{array}$ & $106 \pm 7.6^{d}$ & NA & \\
\hline Intra-AAT $\left(\mathrm{cm}^{3}\right)^{\mathrm{e}}$ & & & 0.58 \\
\hline TFA $(n=19)$ & $133 \pm 9$ & $141 \pm 10$ & \\
\hline Control $(n=19)$ & $135 \pm 14$ & $146 \pm 17$ & \\
\hline $\begin{array}{l}\text { Lean reference subjects } \\
(n=19)\end{array}$ & $33 \pm 4^{d}$ & NA & \\
\hline Intra/subcutaneous $A A T^{\mathrm{e}, \mathrm{f}}$ & & & 0.67 \\
\hline TFA $(n=19)$ & $0.45 \pm 0.04$ & $0.46 \pm 0.05$ & \\
\hline Control $(n=19)$ & $0.56 \pm 0.08$ & $0.60 \pm 0.10$ & \\
\hline $\begin{array}{l}\text { Lean reference subjects } \\
(n=19)\end{array}$ & $0.31 \pm 0.03^{d}$ & NA & \\
\hline Liver fat $(\%)^{f}$ & & & 0.87 \\
\hline TFA $(n=23)$ & $2.7(1.3 ; 5.7)$ & $1.9(0.8 ; 4.6)$ & \\
\hline Control $(n=23)$ & $2.4(1.0 ; 5.6)$ & $1.8(0.6 ; 5.1)$ & \\
\hline $\begin{array}{l}\text { Lean reference subjects } \\
(n=19)\end{array}$ & $0.1(0.07 ; 0.3)^{d}$ & NA & \\
\hline
\end{tabular}

Abbreviations: AAT, abdominal adipose tissue; ANCOVA, analysis of covariance; NA, not applicable; TFA, trans fatty acid. ${ }^{\mathrm{a} V a l u e s}$ are means \pm s.e.m. or geometric means (95\% confidence interval) for skewed data, completers only. ${ }^{\text {b}}$ There were no significant differences in baseline values between diet groups (unpaired $t$-test). ${ }^{C} P$ value refers to differences between diet groups at week 16 with week 0 as a covariate, by ANCOVA. Adjusting for weight change did not affect the results. ${ }^{\mathrm{d}}$ Significantly different from overweight intervention subjects (diet groups combined) by unpaired $t$-test or Kruskal-Wallis test for skewed data; $P<0.05$. ${ }^{e}$ Obtained from MR imaging. ${ }^{f}$ ANCOVA performed on log10-transformed values.

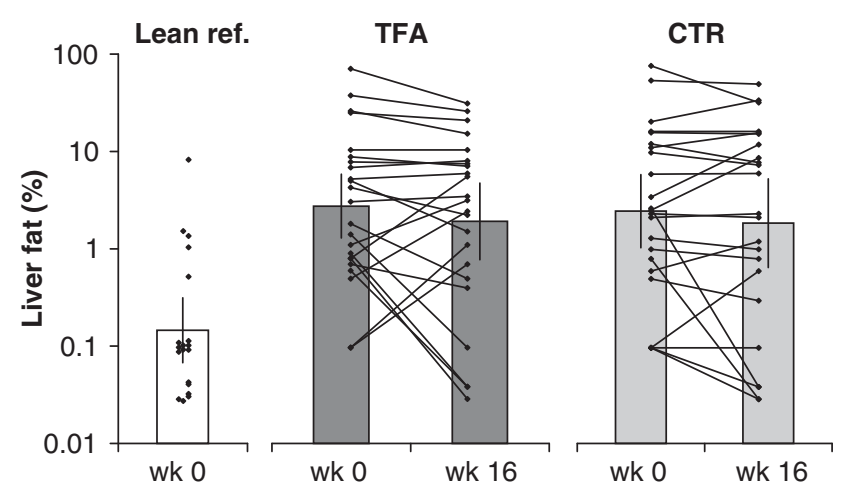

Figure 4 Liver fat percentage in the trans fat group (TFA; $n=23$ ) and in the control group (CTR; $n=23$ ) before and after 16 weeks of dietary intervention and in lean references $(n=19)$ obtained by ${ }^{1} \mathrm{H}$ MR spectroscopy. Bars show geometric means ( $95 \%$ confidence intervals). There was no significant effect of diet, $P=0.87$ for differences between diet groups at week 16 with week 0 as a covariate, by analysis of covariance performed on log-transformed data. The liver fat $\%$ in lean references was significantly lower than in overweight intervention subjects (diet groups combined) by Kruskal-Wallis test for skewed data; $P<0.01$.

produced TFA from food products sold in Denmark, the study population was depleted of industrially produced TFA. In metabolically vulnerable women, the present intervention study found that a high intake of TFA from partially hydrogenated soybean oil for 16 weeks increased the ratio of plasma LDL- to HDL-C, but had no detectable effect on hepatic and abdominal fat deposition.

In dietary interventions examining the effect of a specific FA composition, it is always a challenge to decide on an appropriate control fat. For the present study, a fat containing predominantly MUFA but also SFA and PUFA was chosen because fast food products and snacks produced without TFA have higher levels of all three FA classes compared with similar products that are high in TFA. ${ }^{17}$ Also, a recent report has documented that when products high in TFA are reformulated to eliminate TFA, the TFA is mainly replaced by unsaturated FA. ${ }^{18}$

TFA intake led to an increase in LDL-C as well as a decrease in HDL-C concentrations. The mean difference between diet groups in the TC/HDL-C ratio of 0.45 was somewhat larger than what could have been expected from a recent metaanalysis by Mozaffarian and Clarke $^{2}$ estimating that a replacement of $1 \mathrm{E} \%$ TFA by SFA, MUFA and PUFA increases the TC/HDL-C ratio by $0.031,0.054$ and 0.067 , respectively. ${ }^{2}$ The present study used a blend of palm oil and high oleic sunflower oil as control fat, whereby $7 \mathrm{E} \%$ from TFA in the TFA group was compared with an intake of $1.6 \mathrm{E} \%$ from SFA, $4.7 \mathrm{E} \%$ from MUFA and $0.7 \mathrm{E} \%$ from PUFA in the control group. According to the estimates from the study by Mozaffarian and Clarke, a difference in the TC/HDL-C ratio of 0.35 between the TFA and control diet was expected. The effect of TFA on blood lipids in the present study is also considerably greater than that observed in our previous 
Table 4 Blood lipids in the TFA $(n=24)$ and control $(n=25)$ groups and in lean reference subjects $(n=19)^{\mathrm{a}}$

\begin{tabular}{|c|c|c|c|c|c|c|}
\hline & \multirow[t]{2}{*}{ Week $0^{\mathrm{b}}$} & \multirow[t]{2}{*}{ Week 8} & \multirow[t]{2}{*}{ Week 16} & \multicolumn{3}{|c|}{ ANCOVA $^{\mathrm{c}}$} \\
\hline & & & & $\mathrm{P}_{\text {time*diet }}$ & $P_{\text {diet }}$ & $\mathrm{P}_{\text {time }}$ \\
\hline Total cholesterol $\left(\mathrm{mmoll}^{-1}\right)$ & & & & 0.16 & 0.03 & 0.88 \\
\hline TFA & $5.44 \pm 0.19$ & $6.07 \pm 0.21$ & $5.95 \pm 0.19$ & & & \\
\hline Control & $5.54 \pm 0.20$ & $5.77 \pm 0.18$ & $5.86 \pm 0.21$ & & & \\
\hline Lean reference subjects & $5.46 \pm 0.18$ & NA & NA & & & \\
\hline HDL cholesterol $\left(\mathrm{mmoll}^{-1}\right)$ & & & & 0.23 & 0.03 & $<0.001$ \\
\hline TFA & $1.54 \pm 0.06$ & $1.54 \pm 0.05$ & $1.38 \pm 0.04$ & & & \\
\hline Control & $1.65 \pm 0.07$ & $1.69 \pm 0.08$ & $1.59 \pm 0.06$ & & & \\
\hline Lean reference subjects & $1.69 \pm 0.05$ & NA & NA & & & \\
\hline LDL cholesterol $\left(\mathrm{mmoll}^{-1}\right)$ & & & & 0.24 & 0.002 & 0.01 \\
\hline TFA & $3.39 \pm 0.18$ & $3.91 \pm 0.19$ & $4.01 \pm 0.18$ & & & \\
\hline Control & $3.36 \pm 0.20$ & $3.46 \pm 0.16$ & $3.72 \pm 0.19$ & & & \\
\hline Lean reference subjects & $3.47 \pm 0.17$ & NA & NA & & & \\
\hline LDL/HDL-cholesterol ratio & & & & 0.23 & $<0.001$ & $<0.001$ \\
\hline TFA & $2.31 \pm 0.16$ & $2.64 \pm 0.17$ & $3.02 \pm 0.20$ & & & \\
\hline Control & $2.20 \pm 0.20$ & $2.20 \pm 0.17$ & $2.45 \pm 0.16$ & & & \\
\hline Lean reference subjects & $2.08 \pm 0.11$ & NA & NA & & & \\
\hline Total/HDL-cholesterol ratio & & & & 0.22 & $<0.001$ & $<0.001$ \\
\hline TFA & $3.65 \pm 0.19$ & $4.08 \pm 0.22$ & $4.45 \pm 0.23$ & & & \\
\hline Control & $3.54 \pm 0.23$ & $3.60 \pm 0.21$ & $3.83 \pm 0.20$ & & & \\
\hline Lean reference subjects & $3.25 \pm 0.12$ & NA & NA & & & \\
\hline Triglycerides $\left(\mathrm{mmoll}^{-1}\right)^{\mathrm{d}}$ & & & & 0.78 & 0.39 & 0.09 \\
\hline TFA & $1.13 \pm 0.10$ & $1.39 \pm 0.15$ & $1.24 \pm 0.09$ & & & \\
\hline Control & $1.19 \pm 0.10$ & $1.35 \pm 0.12$ & $1.23 \pm 0.11$ & & & \\
\hline Lean reference subjects & $0.67 \pm 0.04^{\mathrm{e}}$ & NA & NA & & & \\
\hline
\end{tabular}

Abbreviations: ANCOVA, analysis of covariance; HDL, high-density lipoprotein; LDL, low-density lipoprotein; NA, not applicable; TFA, trans fatty acid. ${ }^{2}$ Values are means \pm s.e.m., completers only. ${ }^{b}$ There were no significant differences in baseline values between diet group (unpaired $t$-test, $P>0.05$ ). ${ }^{c} P$ values were derived by repeated-measures analysis of covariance testing for interaction between diet group and time (week 8,16 ) with week 0 as a covariate. $P$ values for effects of diet and time, respectively, were derived from analyses omitting the interaction term. ${ }^{\mathrm{d}}$ ANCOVA performed on log 10 -transformed values. ${ }^{\mathrm{e}}$ Significantly different from overweight intervention subjects (diet groups combined) by unpaired $t$-test; $P<0.05$.

8-week study with a similar design but with normal-weight young men. ${ }^{19}$ The fact that only moderately overweight women above the age of 45 years were enrolled may help to explain the more pronounced effect of TFA on plasma cholesterol in the present study. It has previously been suggested that a high TFA intake may be more detrimental for women than for men. ${ }^{20,21}$

The TC/HDL-C ratio is twice as informative of cardiovascular risk as TC or LDL-C alone. ${ }^{3}$ The increase in the TC/HDL-C ratio in the TFA group of 0.45 compared with the control subjects may translate into an increase in the risk of coronary heart disease of approximately $20 \% .^{3}$ An intake of $15 \mathrm{~g} \mathrm{day}^{-1}$ of TFA may be a likely scenario in selected communities or subpopulations around the world. For example, in an Iranian population, the mean TFA intake was as high as $12.3 \mathrm{~g} \mathrm{day}^{-1}$ in $2001-2003,{ }^{22}$ and in 1989-91 the mean TFA intake in the US population was $5.3 \mathrm{~g} \mathrm{day}^{-1}$ and $10 \%$ of the population was estimated to have an intake above $9.4 \mathrm{~g} \mathrm{day}^{-1} \cdot{ }^{23}$ However, although more recent intake estimates are lacking, the TFA intake in the United States has most likely declined in the recent years as increased trans fat awareness has forced the food industry to limit the content of TFA. ${ }^{24}$

In prospective cohort studies, TFA intake has been positively associated with changes in weight and waist circumference. ${ }^{5,6}$ In our study, women in both diet groups experienced slight weight gains during the course of the intervention, implying that they did not succeed in isocalorically substituting the test food for food items from their habitual diets. We observed weak trends toward greater increases in waist circumference and total fat mass in the TFA group compared with controls, independently of body weight changes. However, these trends were not reflected by the observed changes in abdominal fat deposition. We saw no differences between the diet groups in either intraabdominal or subcutaneous abdominal fat deposition, nor in the ratio between the two. Thereby, the present study could not substantiate the findings from a study in green monkeys observing an increase in the ratio of intra- to subcutaneous AAT volume irrespective of weight gain after 6 years of isocaloric TFA feeding. ${ }^{7}$ It is likely that 16 weeks of exposure was too short a period of time to detect changes in 
abdominal fat deposition independent of body weight even with high daily TFA intakes. Indeed, body weight gain may be an intermediary step leading to TFA-induced increases in abdominal fat deposition, which we may not have been able to detect with our isocaloric study design. However, in comparison, conjugated linoleic acid supplementation of $4.5 \mathrm{~g} \mathrm{day}^{-1}$ has been shown to significantly decrease the fat mass (assessed by DXA) in postmenopausal women within 16 weeks on isocaloric diets. ${ }^{25}$ Also, isocaloric diets high in PUFA have been found to significantly decrease subcutaneous AT (assessed by single-slice MRI) compared with diets high in SFA within 5 weeks, despite no changes in body weight. $^{26}$ This confirms that abdominal fat distribution can be affected by dietary fat manipulations within the timeframe of our study.

As a long-term dietary intervention study providing high daily amounts of TFA is not feasible, it is questionable whether an effect on abdominal fat will ever be established in humans. Conversely, potential changes in liver fat due to high intakes of TFA might be detected within a reasonable period of time. Liver fat is suggested to be a more important risk factor for obesity-related metabolic disorders than intra-AAT. $^{27}$

We are the first to report on the influence of a high TFA intake on liver fat deposition in humans. The data from the present study were consistent with liver fat being from $61 \%$ lower to $140 \%$ higher in the TFA group at week 16 when compared with controls. Given the geometric mean liver fat concentration of $\sim 2.5 \%$ at baseline, the confidence limits may translate into an absolute mean decrease in liver fat of $1.5 \%$ or an absolute increase of $3.5 \%$. Therefore, our results cannot discount the possibility that TFA intake may exert effects on liver fat smaller than this, prohibiting firm conclusions on minor changes. However, no detectable trend in liver fat was observed in either direction in either diet group and plasma liver enzymes remained unchanged, indicating that if TFA induced changes in liver fat concentration, they were small indeed.

In several rodent studies TFA feeding has been shown to increase liver fat by more than $100 \%$ compared with animals fed cis FA, ${ }^{8,9,28}$ although this is not a consistent finding. ${ }^{29,30}$ Yet, extrapolation from animal studies is problematic. Although it has been documented that liver fat deposition in humans is affected by intake of energy and fat (liver fat increased by $100 \%$ (from 2 to $4 \%$ and from 11 to $22 \%$ ) after 3-4 days of hypercaloric high-fat diets ${ }^{31,32}$ and by $35 \%$ (from 10 to $14 \%$ ) after 14 days of eucaloric high-fat diets ${ }^{33}$ ), the influence of dietary FA composition on liver fat deposition in humans has yet to be evaluated in controlled trials. It has, however, been documented that intake of specific FAs can affect liver fat accumulation; consumption of n-3 long chain PUFA supplements $\left(2-4 \mathrm{~g} \mathrm{day}^{-1}\right)$ for $8-26$ weeks has been shown to reduce liver fat in subjects with hepatic steatosis. ${ }^{34,35}$

Also, liver fat is affected by body weight changes and has been shown to decrease by $30-60 \%$ in obese subjects after $\sim 6-11 \mathrm{~kg}$ weight loss. ${ }^{36,37}$ In accordance, we observed significant decreases of about $40 \%$ in liver fat mean values after a 12-week dietary weight loss period (mean weight loss $\sim 4 \mathrm{~kg}$ ) subsequent to the dietary intervention (data not shown).

It is a strength of the present study that compliance with the study diets was high, as reflected by the magnitude of changes in plasma cholesterol concentrations. However, it may be considered a limitation that the remaining part of the participants' diets was not strictly controlled. Results of 3-day food records collected in the last week of the intervention were not indicative of significant differences between diet groups in intake of either energy or any of the macronutrients (Supplementary Table 2). Yet, we acknowledge that minor differences may not have been picked up by this dietary assessment.

It may also be considered a limitation that AAT volumes were assessed by single-slice MRI, and not by examination of the entire abdominal region. We obtained images at the level of the third lumbar vertebra, which has been shown to be the single-slice volume to correlate best with the intra-AAT of the whole abdominal region in women $\left(R^{2}=0.94-0.97\right){ }^{38-40}$ However, single-slice methods may be less likely to detect small changes in abdominal adiposity. ${ }^{39,41}$

We measured liver fat by ${ }^{1} \mathrm{H}$ MR spectroscopy, which is the most accurate non-invasive technique available. ${ }^{42}$ We aimed to standardize the liver fat assessments by controlling alcohol intake, physical activity and diet from the evening before the examination. However, the fact that the diets were not controlled for longer periods before the scans may have limited our ability to detect small TFA-induced changes in liver fat. Also, as seen in Figure 4, the between-subject variability in baseline liver fat was large (range $0.1-73.0 \%$ ), and it is questionable whether subjects with very high liver fat content will be susceptible to further increases due to dietary manipulations. In future studies, a screening for liver fat content should be considered, since a modest diet-induced change may be more easily detected in a study population with more homogeneous liver fat levels.

In conclusion, our research confirms that part of the increase in risk of cardiovascular disease seen with increased TFA consumption in observational studies is likely to be due to dyslipidemia. The magnitude of the observed impact of TFA intake on blood cholesterol levels suggests that older and overweight populations may be more susceptible to TFA-induced metabolic changes than young and lean ones. Conversely, weight gain-independent induction of liver or intra-abdominal fat deposition could not be identified as a contributory explanatory mechanism.

\section{Conflict of interest}

The authors declare no conflict of interest. 


\section{Acknowledgements}

This work was carried out as a part of the research program of the Danish Obesity Research Centre (DanORC, see http:// www.danorc.dk). DanORC is supported by the Danish Council for Strategic Research (Grant 2101-06-0005). This study was also supported by the Danish Council for Independent Research | Medical Sciences (Grant 271-080715), the Danish Diabetes Association and intramural USDA-ARS CRIS 5306-51530-019-00D. The test fats were kindly provided by AarhusKarlshamn, Denmark.

\section{References}

1 Mozaffarian D, Katan MB, Ascherio A, Stampfer MJ, Willett WC. Trans fatty acids and cardiovascular disease. N Engl J Med 2006; 354: 1601-1613.

2 Mozaffarian D, Clarke R. Quantitative effects on cardiovascular risk factors and coronary heart disease risk of replacing partially hydrogenated vegetable oils with other fats and oils. Eur J Clin Nutr 2009; 63: S22-S33.

3 Lewington S, Whitlock G, Clarke R, Sherliker P, Emberson J, Halsey $\mathrm{J}$ et al. Blood cholesterol and vascular mortality by age, sex, and blood pressure: a meta-analysis of individual data from 61 prospective studies with 55000 vascular deaths. Lancet 2007; 370: 1829-1839.

4 Alberti KGMM, Zimmet P, Shaw J. Metabolic syndrome-a new world-wide definition. A consensus statement from the international diabetes federation. Diabet Med 2006; 23: 469-480.

5 Koh-Banerjee P, Chu NF, Spiegelman D, Rosner B, Colditz G, Willett W et al. Prospective study of the association of changes in dietary intake, physical activity, alcohol consumption, and smoking with 9-y gain in waist circumference among 16587 US men. Am J Clin Nutr 2003; 78: 719-727.

6 Field AE, Willett WC, Lissner L, Colditz GA. Dietary fat and weight gain among women in the nurses/' health study[ast]. Obesity 2007; 15: 967-976.

7 Kavanagh K, Jones KL, Sawyer J, Kelley K, Carr JJ, Wagner JD et al. Trans fat diet induces abdominal obesity and changes in insulin sensitivity in monkeys. Obesity 2007; 15: 1675-1684.

8 Colandre ME, Diez RS, Bernal CA. Metabolic effects of trans fatty acids on an experimental dietary model. Br J Nutr 2003; 89: 631-638.

9 Dorfman SE, Laurent D, Gounarides JS, Li X, Mullarkey TL, Rocheford EC et al. Metabolic implications of dietary trans-fatty acids. Obesity 2009; 17: 1200-1207.

10 Baecke J, Burema J, Frijters J. A short questionnaire for the measurement of habitual physical activity in epidemiological studies. Am J Clin Nutr 1982; 36: 936-942.

11 Christie WW.. Lipid Analysis : Isolation, Separation, Identification, and Structural Analysis of Lipids, 3rd edn. The Oily Press: Bridgwater, 2003.

12 Møller A, Saxholt E, Christensen AT, Hartkopp HB, Hess Ygil K. Danish Food Composition Databank, revision 6.0. Food Informatics, Institute of Food Safety and Nutrition, Danish Institute for Food and Veterinary Research: Copenhagen, Denmark. http:// www.foodcomp.dk/ 2005.

13 Mehta SR, Thomas EL, Bell JD, Johnston DG, Taylor-Robinson SD. Non-invasive means of measuring hepatic fat content. World $J$ Gastroenterol 2008; 14: 3476-3483.

14 Friedewald WT, Levy RI, Fredrickson DS. Estimation of the concentration of low-density lipoprotein cholesterol in plasma, without use of the preparative ultracentrifuge. Clin Chem 1972; 18: 499-502.
15 Ascherio A, Katan MB, Zock PL, Stampfer MJ, Willett WC. Trans fatty acids and coronary heart disease. N Engl J Med 1999; 340: 1994-1998.

16 Westerbacka J, Corner A, Tiikkainen M, Tamminen M, Vehkavaara S, Hakkinen AM et al. Women and men have similar amounts of liver and intra-abdominal fat, despite more subcutaneous fat in women: implications for sex differences in markers of cardiovascular risk. Diabetologia 2004; 47: 1360-1369.

17 Stender S, Astrup A, Dyerberg J. What went in when trans went out? N Engl J Med 2009; 361: 314-316.

18 Ratnayake WMN, L'Abbe MR, Mozaffarian D. Nationwide product reformulations to reduce trans fatty acids in Canada: when trans fat goes out, what goes in? Eur J Clin Nutr 2009; 63: 808-811.

19 Dyerberg J, Eskesen DC, Andersen PW, Astrup A, Buemann B, Christensen $\mathrm{JH}$ et al. Effects of trans- and n-3 unsaturated fatty acids on cardiovascular risk markers in healthy males. An 8 weeks dietary intervention study. Eur J Clin Nutr 2004; 58: 1062-1070.

20 Chardigny JM, Destaillats F, Malpuech-Brugere C, Moulin J, Bauman DE, Lock AL et al. Do trans fatty acids from industrially produced sources and from natural sources have the same effect on cardiovascular disease risk factors in healthy subjects? Results of the trans Fatty Acids Collaboration (TRANSFACT) study. Am J Clin Nutr 2008; 87: 558-566.

$21 \mathrm{Hu}$ FB, Stampfer MJ, Manson JE, Rimm E, Colditz GA, Rosner BA et al. Dietary fat intake and the risk of coronary heart disease in women. N Engl J Med 1997; 337: 1491-1499.

22 Mozaffarian D, Abdollahi M, Campos H, HoushiarRad A, Willett WC. Consumption of trans fats and estimated effects on coronary heart disease in Iran. Eur J Clin Nutr 2007; 61: 1004-1010.

23 Allison DB, Egan S, Barraj L, Caughman C, Infante M, Heimbach J. Estimated intakes of trans fatty and other fatty acids in the US population. J Am Diet Assoc 1999; 99: 166-174.

24 L'Abbe MR, Stender S, Skeaff CM, Ghafoorunissa, Tavella M. Approaches to removing trans fats from the food supply in industrialized and developing countries. Eur J Clin Nutr 2009; 63: S50-S67.

25 Raff M, Tholstrup T, Toubro S, Bruun JM, Lund P, Straarup EM et al. Conjugated linoleic acids reduce body fat in healthy postmenopausal women. J Nutr 2009; 139: 1347-1352.

26 Summers LKM, Fielding BA, Bradshaw HA, Ilic V, Beysen C, Clark ML et al. Substituting dietary saturated fat with polyunsaturated fat changes abdominal fat distribution and improves insulin sensitivity. Diabetologia 2002; 45: 369-377.

27 Fabbrini E, Magkos F, Mohammed BS, Pietka T, Abumrad NA, Patterson BW et al. Intrahepatic fat, not visceral fat, is linked with metabolic complications of obesity. Proc Natl Acad Sci USA 2009; 106: 15430-15435.

28 Tetri LH, Basaranoglu M, Brunt EM, Yerian LM, NeuschwanderTeri BA. Severe NAFLD with hepatic necroinflammatory changes in mice fed trans fats and a high-fructose corn syrup equivalent. Am J Physiol Gastrointest Liver Physiol 2008; 295: 987-995.

29 Giudetti AM, Beynen AC, Lemmens AG, Gnoni GV, Geelen MJH. Hepatic fatty acid metabolism in rats fed diets with different contents of C18:0, C18:1cis and C18:1trans isomers. Br J Nutr 2003; 90: 887-893.

30 Koppe SWP, Elias M, Moseley RH, Green RM. Trans fat feeding results in higher serum alanine aminotransferase and increased insulin resistance compared with a standard murine high-fat diet. Am J Physiol Gastrointest Liver Physiol 2009; 297: G378-G384.

31 Bortolotti M, Kreis R, Debard C, Cariou B, Faeh D, Chetiveaux M et al. High protein intake reduces intrahepatocellular lipid deposition in humans. Am J Clin Nutr 2009; 90: 1002-1010.

32 van der Meer RW, Hammer S, Lamb HJ, Frolich M, Diamant M, Rijzewijk LJ et al. Effects of short-term high-fat, high-energy diet on hepatic and myocardial triglyceride content in healthy men. J Clin Endocrinol Metab 2008; 93/7: 2702-2708. 
33 Westerbacka J, Lammi K, Hakkinen AM, Rissanen A, Salminen I, Aro A et al. Dietary fat content modifies liver fat in overweight nondiabetic subjects. J Clin Endocrinol Metab 2005; 90/5: 2804-2809.

34 Cussons AJ, Watts GF, Mori TA, Stuckey BGA. Omega-3 fatty acid supplementation decreases liver fat content in polycystic ovary syndrome: a randomized controlled trial employing proton magnetic resonance spectroscopy. J Clin Endocrinol Metab 2009; 94/10: 3842-3848.

35 Spadaro L, Magliocco O, Spampinato D, Piro S, Oliveri C, Alagona $\mathrm{C}$ et al. Effects of n-3 polyunsaturated fatty acids in subjects with nonalcoholic fatty liver disease. Digest Liver Dis 2008; 40: 194-199.

36 Viljanen APM, Iozzo P, Borra R, Kankaanpaa M, Karmi A, Lautamaki $\mathrm{R}$ et al. Effect of weight loss on liver free fatty acid uptake and hepatic insulin resistance. J Clin Endocrinol Metab 2009; 94: 50-55.

37 Sato F, Tamura Y, Watada H, Kumashiro N, Igarashi Y, Uchino H et al. Effects of diet-induced moderate weight reduction on intrahepatic and intramyocellular triglycerides and glucose metabolism in obese subjects. J Clin Endocrinol Metab 2007; 92: 3326-3329.

38 Han TS, Kelly IE, Walsh K, Greene RME, Lean MEJ. Relationship between volumes and areas from single transverse scans of intra-abdominal fat measured by magnetic resonance imaging. Int J Obes 1997; 21: 1161-1166.

39 Shen W, Punyanitya M, Wang Z, Gallagher D, St-Onge MP, Albu J et al. Visceral adipose tissue: relations between single-slice areas and total volume. Am J Clin Nutr 2004; 80: 271-278.

40 Demerath EW, Shen W, Lee M, Choh AC, Czerwinski SA, Siervogel RM et al. Approximation of total visceral adipose tissue with a single magnetic resonance image. Am J Clin Nutr 2007; 85: 362-368.

41 Thomas EL, Bell JD. Influence of undersampling on magnetic resonance imaging measurements of intra-abdominal adipose tissue. Int J Obes Relat Metab Disord 2003; 27: 211-218.

42 van Werven JR, Hoogduin JM, Nederveen AJ, van Vliet AA, Wajs E, Vandenberk P et al. Reproducibility of 3.0 Tesla magnetic resonance spectroscopy for measuring hepatic fat content. J Magn Reson Imaging 2009; 30: 444-448.

This work is licensed under the Creative Commons Attribution-NonCommercial-No Derivative Works 3.0 Unported License. To view a copy of this license, visit http://creativecommons.org/ licenses/by-nc-nd/3.0/

Supplementary Information accompanies the paper on the Nutrition and Diabetes website (http://www.nature.com/nutd) 